\title{
Investigation of the Mode Structures of Multiphoton Induced Ultraviolet Laser in a ZnO Microrod
}

\author{
Guangping Zhu \\ School of Physics and Electronic Information, Huaibei Normal University, Huaibei 235000, China \\ Correspondence should be addressed to Guangping Zhu; gpzhu@chnu.edu.cn
}

Received 9 November 2016; Accepted 21 February 2017; Published 20 March 2017

Academic Editor: Baofu Ding

Copyright (C) 2017 Guangping Zhu. This is an open access article distributed under the Creative Commons Attribution License, which permits unrestricted use, distribution, and reproduction in any medium, provided the original work is properly cited.

Hexagonal wurtzite structural $\mathrm{ZnO}$ microrods were fabricated by vapor-phase transport method. Under the excitation of a pulse laser with $1200 \mathrm{~nm}$ wavelength, the multiphoton induced ultraviolet (UV) laser was observed in a microrod. The dependence of the laser mode structures on pump intensity was investigated. The result indicates that the laser belongs to whispering gallery mode (WGM) at low pump intensity and Fabry-Perot (FP) mode at high pump intensity. The corresponding positive feedback mechanisms were discussed.

\section{Introduction}

$\mathrm{ZnO}$ is a promising candidate for developing high efficiency ultraviolet optic-electronic devices operating at room temperature due to its wide band-gap and large exciton binding energy. Recent research progress in the fabrication of micro/ nanostructured $\mathrm{ZnO}$ has created opportunities for developing micron-sized or nanosized optoelectronic devices. Optically pumped lasers have been achieved from various $\mathrm{ZnO}$ micro/nanostructures, such as thin films [1], powders [2], microtubes [3], nano/microwires [4-6], nanobelts [7], nanonails [8], microspheres [9], and nano/microdisks [10, 11] by single-photon absorption in which $\mathrm{ZnO}$ serves as both active gain medium and optical resonant cavity. On the other hand, multiphoton induced laser has also exhibited fascinating interest owing to high spatial resolution and large penetration depth of pump light. For $350 \mathrm{~nm}$ pump light, the single-photon absorption coefficient of $\mathrm{ZnO}$ attains $10^{-5} \mathrm{~cm}^{-1}$, and the corresponding penetration depth is only $100 \mathrm{~nm}$ [12]. Therefore, the pump energy is mainly absorbed near the crystal surface, which limits largely the material availability. On the contrary, $\mathrm{ZnO}$ sample can be fully pumped in multiphoton excitation form by visible or infrared light due to high transmission.

At present, multiphoton induced laser in $\mathrm{ZnO}$ micro/ nanostructures has aroused research interest due to a large nonlinear polarization coefficient. Two-photon and three-photon induced lasers have been reported in $\mathrm{ZnO}$ microneedle [13], nanodisk [14], and nanonail [15]. However, the report about the mode structure change with pump intensity has not been found. In this letter, three-photon induced ultraviolet laser is obtained in a $\mathrm{ZnO}$ microrod under excitation of $1200 \mathrm{~nm}$ infrared laser, and the dependence of mode structure on pump intensity will be discussed in detail.

\section{Experiment}

$\mathrm{ZnO}$ microrods were prepared by a simple vapor-phase transport method similar to our previous work [16]. The mixture of high pure zinc oxide and graphite powders was put at an end of a two-end-open quartz tube $(2 \mathrm{~cm}$ diameter and $24 \mathrm{~cm}$ length) as source materials, and a silicon wafer was placed next to source materials as substrate. Afterwards, the quartz tube was put into a horizontal tube furnace. The reaction temperature of source material was set up at $1130^{\circ} \mathrm{C}$ and the reaction time was $30 \mathrm{~min}$.

A femtosecond pulse laser with $1200 \mathrm{~nm}$ wavelength from an optical parametric oscillator (OPO) (repetition frequency $1000 \mathrm{~Hz}$, pulse duration $150 \mathrm{fs}$ ) was focused into an $\sim 100 \mu \mathrm{m}$ diameter light spot onto the $\mathrm{ZnO}$ microrod sides. The output spectra were recorded by a fiber coupled optical multichannel analyzer (OMA) at room temperature. 


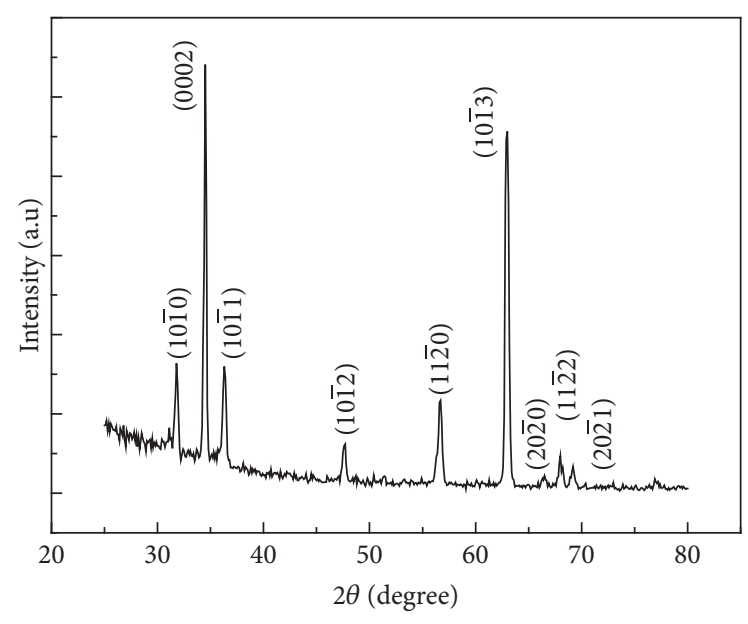

(a)

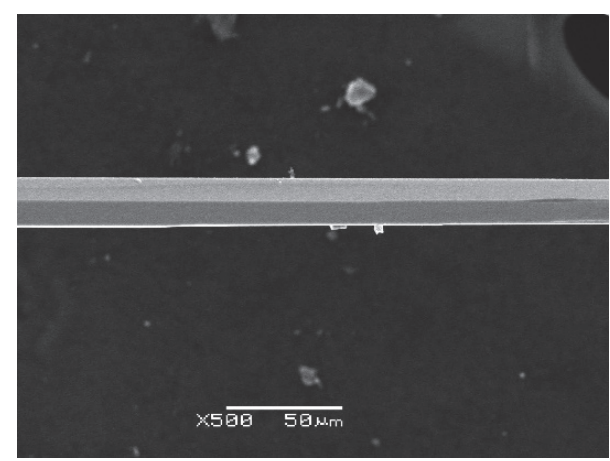

(b)

FIGURE 1: (a) The XRD pattern of the $\mathrm{ZnO}$ microrod and (b) the SEM image of the selected microrods for laser investigation.

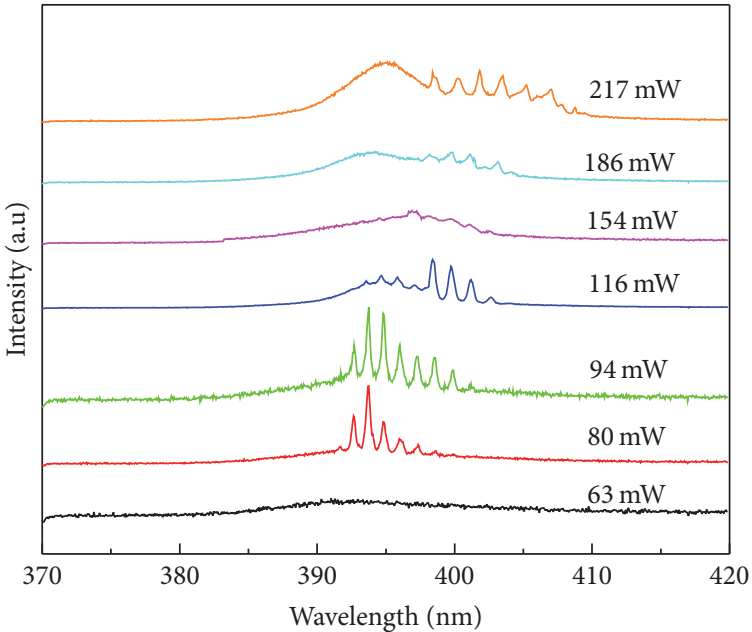

(a)

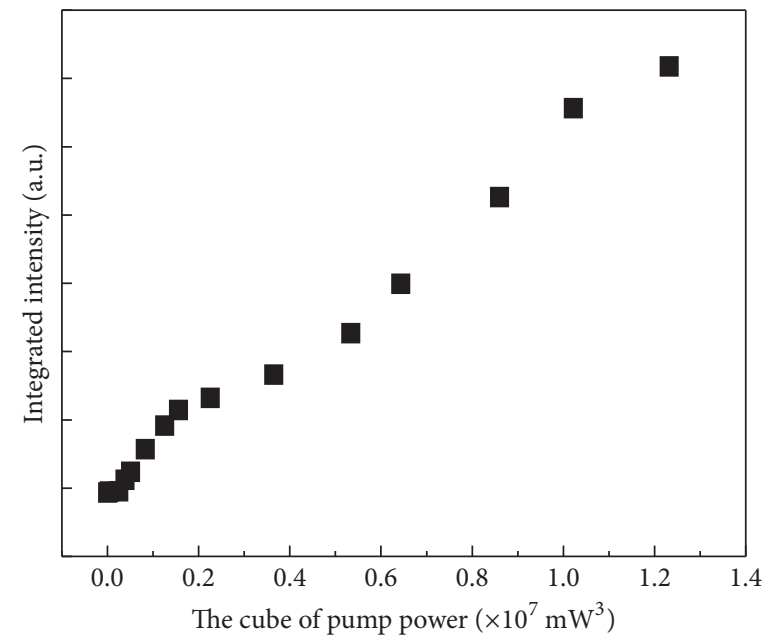

(b)

Figure 2: (a) The emission spectra at different pump power and (b) the variation of the spectrum integrated intensity with the cube of pump emission.

\section{Result and Discussion}

The XRD pattern of $\mathrm{ZnO}$ microrods is shown in Figure 1(a). All diffraction peaks in the figure match the crystal plane indexes of hexagonal wurtzite structural $\mathrm{ZnO}$ with lattice constants of $a=0.325 \mathrm{~nm}$ and $c=0.521 \mathrm{~nm}$. The narrow diffraction peaks imply a high crystalline quality of the $\mathrm{ZnO}$ products. Figure 1(b) exhibits the SEM image of the selected microrod for laser investigation, which has a $\sim 19 \mu \mathrm{m}$ diagonal diameter.

Figure 2(a) shows the emission spectra at different pump power. At lower pump power of $63 \mathrm{~mW}$, the spectrum exhibits a typical spontaneous emission band centered at $392 \mathrm{~nm}$. When the pump power attains $80 \mathrm{~mW}$, some narrow peaks appear in a range from $391 \mathrm{~nm}$ to $398 \mathrm{~nm}$ on the broad UV band, which indicates a lasing process occurring in the $\mathrm{ZnO}$ microrod. Under $94 \mathrm{~mW}$ pump power, the emission peaks are narrower and more peaks emerged in long wavelength edge. It is clearly that the UV lasers contain both exciton emission and electron-hole plasma (EHP) emission [17] at above lower excitation intensity. With pump power further increased to $116 \mathrm{~mW}$, the exciton laser nearly disappeared, and only the EHP emissions exist in the laser modes. When the pump power attains $154 \mathrm{~mW}$, no obvious laser modes can be observed. However, when the pump power rises to $186 \mathrm{~mW}$, the laser modes appear once again, and with pump power increase, there are more modes in long wavelength edge. It is noted that the laser mode spacing obviously changes with the pump power increase. At lower excitation level, for example, $94 \mathrm{~mW}$, although the mode spacing slightly varies with wavelength, the average mode spacing is only about $1.1 \mathrm{~nm}$. However, at a higher pump power of $217 \mathrm{~mW}$, the average mode spacing attains about $1.66 \mathrm{~nm}$. In our experiment, the excitation wavelength is $1200 \mathrm{~nm}$, and the 
laser wavelength is about $400 \mathrm{~nm}$. It can be believed that the lasing is generated by three-photon absorption. Figure 2(b) shows the curve of the emission intensity versus the cube of pump power. From the figure, two distinctly different lasing regions can be observed, one showing lower threshold and larger slope, whereas the other shows high threshold and small slope. The above discussion implies that the laser mechanism changes with pump intensity.

It has been known that the laser in $\mathrm{ZnO}$ crystal may be attributed to three types $[2,4,5]$ : (a) random laser, (b) F$P$ laser, and (c) whispering gallery mode (WGM) laser. The random cavity model is put forward to explain the lasing in disordered $\mathrm{ZnO}$ mediums, which is attributed to recurrent scattering by localized photons. F-P resonant cavity model is introduced to interpret the laser modes in single wire/rodlike micro/nanostructure, in which two smooth end facets serve as cavity mirrors. For $c$-axis symmetrical hexagonal $\mathrm{ZnO}$ nanostructures, such as micro/nanodisks and microrods, the lasing mechanism can also result from whispering gallery cavity, in which the light wave can propagate circularly due to multiple total internal reflection caused by the six sides. In the three mechanisms, random cavity and F-P cavity cannot provide a high level of light confinement due to strong scattering and the large transmission, which result in a relatively low quality factor. On the other hand, WGM laser based on total internal reflection has unique properties, including low loss, narrow linewidth, and high quality factor.

It is obvious that the random laser cannot be generated in the $\mathrm{ZnO}$ microrod. Supposing that the laser originates from F-P cavity formed by two end facets of the microrod, and ignoring the variation of refractive index with wavelength, the laser mode spacing can be calculated according to the following equation:

$$
\Delta \lambda=\frac{\lambda^{2}}{2 n L} .
$$

Here $\lambda, n$ are wavelength and refractive index corresponding to some mode, and $L$ is the length of the microrod. In our experiment, the microrod length is in magnitude order of millimeter and $n$ is about 2.2 for $395 \mathrm{~nm}$ center wavelength; thus it can be estimated that the mode spacing is about $0.04 \mathrm{~nm}$, which is far less than the experiment value, so the laser cannot also originate from F-P cavity formed by two end facets. Due to the high relative refractive index of $\mathrm{ZnO}$ to air for UV light, the six sides of the microrod can also construct the positive feedback cavity, which includes (1) F$\mathrm{P}$ cavity in which the light bounces forth and back between the two opposite faces and (2) WGM cavity in which the light is fully reflected sequentially by the six sides of the microrod with a $60^{\circ}$ incident angle. In order to further explain the positive feedback mechanism, the mode spacing of the laser for WG cavity and F-P cavity will be calculated in the following section, respectively.

For WGM laser, the resonant conditions of TE (the electric field is perpendicular to the symmetry axis of the microrod) and TM modes (the electric field is parallel to the symmetry axis of the microrod) can be deduced by Fresnel formula [13], which is expressed as follows:

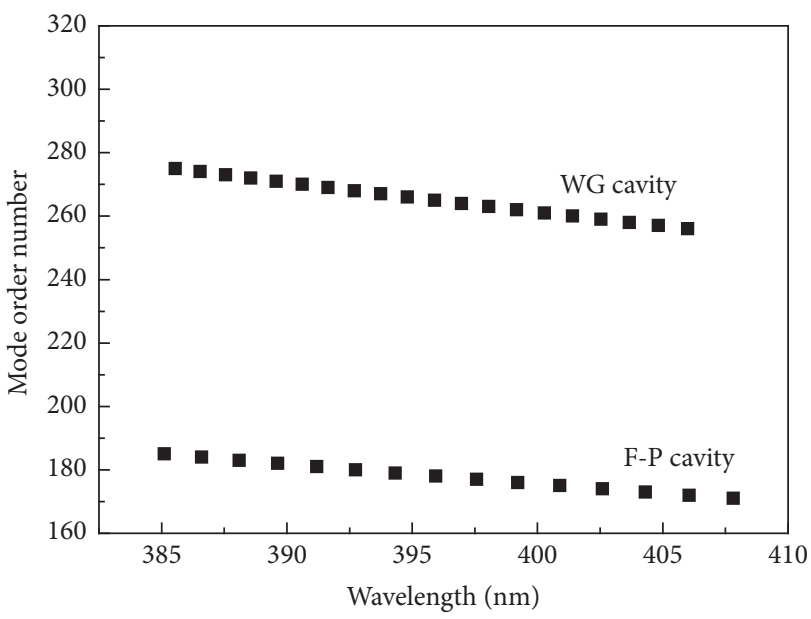

FIGURE 3: The calculation curve of mode order number versus wavelength in the case of F-P and WG cavity.

$$
n L=\lambda\left(N+\frac{6}{\pi} \tan ^{-1} \beta \sqrt{4 n^{2}-3}\right) .
$$

In (2), $N$ is mode order number, $L$ the path length of a light circulation, and $\beta$ factor depends on the laser polarization, $\beta=n$ for TE polarization and $\beta=1 / n$ for TM polarization. For F-P cavity laser, according to standing wave condition, the mode equation can be deduced as follows:

$$
N \frac{\lambda}{2}=n L,
$$

where $L$ is the distance of the two opposite sides.

As a birefringent crystal, the refractive index of the wurtzite structural $\mathrm{ZnO}$ depends on the polarization. For TE and TM polarization, the refractive index can be expressed, respectively, as follows [18]:

$$
\begin{aligned}
n_{\mathrm{TE}}= & 1.916+1.145 \times 10^{-2}(h \nu)^{2}+1.6507 \\
& \times 10^{-3}(h \nu)^{4} \\
n_{\mathrm{TM}}= & 1.9384+1.1775 \times 10^{-2}(h \nu)^{2}+1.5237 \\
& \times 10^{-3}(h \nu)^{4} .
\end{aligned}
$$

The unit of $h v$ is $\mathrm{eV}$ in (4).

The mode order numbers and corresponding wavelengths of TE and TM mode for WGM cavity and F-P cavity can be calculated by (2) and (4) or (3) and (4). According to Nobis et al. reports $[18,19]$, in needle-like or rod-like $\mathrm{ZnO}$ nanostructures, TM modes are main contents of WGM laser. So the modes observed should be TM modes in the experiment if the lasers originate from WG cavity. In our case, $L$ is about $48 \mu \mathrm{m}$ for WG cavity, the variation of the TM mode order number with wavelength can be calculated as shown in Figure 3. For F-P cavity, $L=16 \mu \mathrm{m}$, it can be deduced that the position of the Nth order TM mode is nearly the same as the $(N-1)$ th TE mode. So, Figure 3 only shows TM mode order number variation with wavelength for F-P cavity. The average 


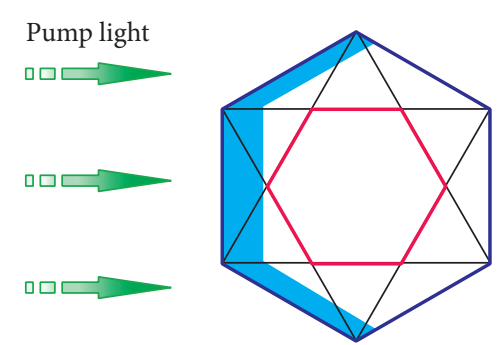

(a)

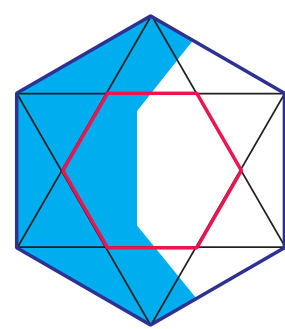

(b)

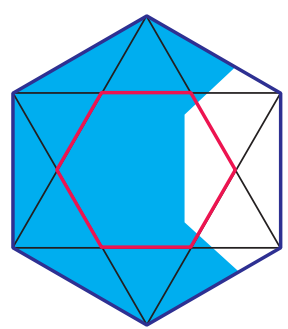

(c)

FIGURE 4: The change of multiphoton absorption region in the microrod with pump power increase: (a) low power; (b) higher power; (c) high power.

mode spacing is about $1.1 \mathrm{~nm}$ for WGM cavity and $1.7 \mathrm{~nm}$ for F-P cavity from Figure 3. Thus, it can be concluded that the lasing should originate from WG cavity at low pump intensity and F-P cavity at high pump intensity.

Based on the above discussion, an interpretation about laser mechanism variation with pump intensity is proposed. It is well known that the multiphoton absorption is highly sensitive to the intensity. In three-photon case, the absorption coefficient is proportional to cube of pump intensity. Figure 4 shows the region change of multiphoton absorption with pump intensity in the microrod, in which the region between the red and the blue hexagon is gain region of WG cavity. At lower pump level, only a thin layer medium near sides of the microrod can obtain enough excitation intensity to generate efficient multiphoton absorption due to the pump light attenuation with incident depth. The thin layer locates the gain region of WG cavity ( $a \sim 4 \mu \mathrm{m}$ depth near the sides of the microrod in our case). Because the loss of WG cavity is far less than that of F-P cavity, the laser will preferentially generate from WG cavity at lower excitation intensity, which explains the reason why the WGM lasers present at lower pump powers of $80 \mathrm{~mW}$ and $94 \mathrm{~mW}$. Due to the depth increase of efficient multiphoton absorption at higher pump power (e.g., $116 \mathrm{~mW}$ ), the multiphoton absorption can occur not only in the region near sides but also in center region. Thus, the luminescence from multiphoton absorption in the center region will deplete inversion population in the WGM gain region by amplified spontaneous emission (ASE), and lead WGM laser weakens. With further increase of pump power, the ASE can quench WGM laser, as is observed at $154 \mathrm{~mW}$ pump power. However, when the excitation intensity increases to a certain threshold once again, it is possible to generate F-P laser formed by two opposite sides because the EHP laser gain is proportional to the difference between EHP density and Mott density, although the F-P cavity loss is higher than WG cavity. It is the reason why the F-P laser can be observed at higher pump power of $186 \mathrm{~mW}$ and $217 \mathrm{~mW}$. Of course, the laser mode spacing will be bigger than that of the WGM laser because the cavity length shortens relative to the WG cavity. It is noted that the exciton emission and EHP emission coexist in laser modes at lower pump intensity. The coexistence of excitonic lasing with the EHP lasing has been previously reported in $\mathrm{ZnO}$ thin film [20], which is attributed to the spatial distribution nonuniformity of the carriers in

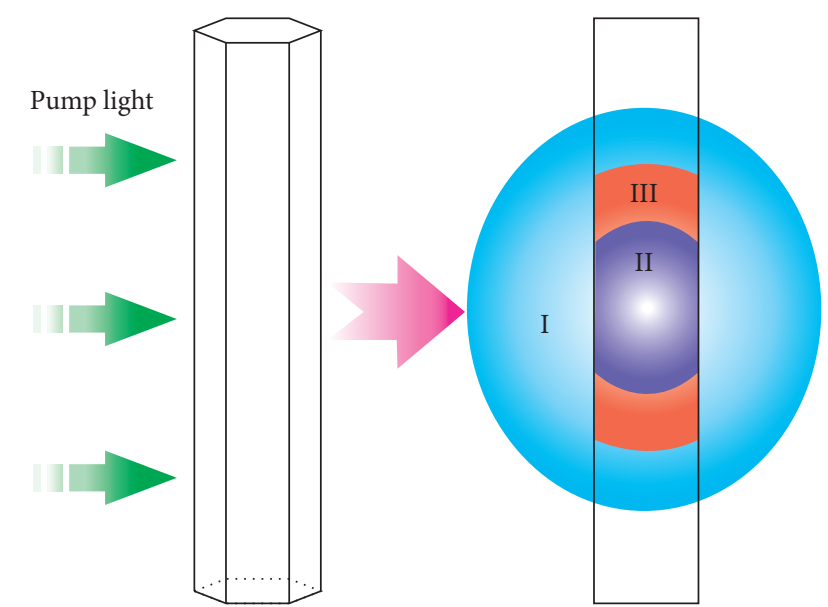

FIGURE 5: The change of carrier type along the microrod length direction: (I) pump light spot; (II) EHP region; (III) exciton region.

the sample. In our case, the coexistence of the two lasers can be attributed to the difference of the carrier density between the center and the edge of the pump light spot along length direction of the microrod (shown in Figure 5), because the pump light is close to Gauss light beam. At lower excitation level, it is possible that the carriers exist in EHP form in center region and in exciton form near edge of the pump spot due to their density difference, which will lead to the EHP laser and excitonic laser in different region. With excitation intensity increase, the EHP region outward spreads. So, the excitonic emission will weaken and the EHP emission enhance, which cause the spectrum red-shift in whole.

In single-photon excitation condition, $\mathrm{ZnO}$ lasing generally originates from the F-P cavity by two end facets of nano/microrod $[1,4]$, or WGM cavity by six sides $[5,18]$. It is difficult to form F-P laser from the two opposite sides. This is due to the fact that the excitation light has a relatively small penetrating depth (about $100 \mathrm{~nm}$ for near-ultraviolet excitation light) and the excited carrier generally locates in WGM laser gain region of the nano/microrod with larger diameter ( $\geq$ laser wavelength). Thus, the laser preferentially generates from WG cavity due to a smaller loss relative to F-P cavity. When the diameter of the rod is smaller than emission wavelength, scattering loss is very high and the WGM laser 
usually cannot be generated. In this case, if the rod length is larger, the F-P cavity by two end facets can still generate enough gain. So, the lasing can be formed by the two end facets.

\section{Conclusion}

In conclusion, the $\mathrm{ZnO}$ microrods with hexagonal crosssection were fabricated by vapor-phase transport process, and an individual microrod was employed to construct a resonant cavity. Under the excitation of the $1200 \mathrm{~nm}$ nanosecond pulse laser, the three-photon excited UV laser was obtained. The result indicates that the lasing resonant mechanism varies with pump intensity. At lower excitation intensity, the exciton emission and EHP emission coexist in laser modes, and the lasers originated from a WGM cavity formed by the six sides of the $\mathrm{ZnO}$ microrod. At higher excitation intensity, the lasers were mainly generated from EHP emission, and the laser positive feedback was provided by F-P cavities by two opposite sides.

\section{Conflicts of Interest}

The author declares that there are no conflicts of interest regarding the publication of this paper.

\section{Acknowledgments}

This work was supported by the Key Foundation of Educational Commission of Anhui Province (KJ2016SD53).

\section{References}

[1] D. M. Bagnall, Y. F. Chen, Z. Zhu, T. Yao, M. Y. Shen, and T. Goto, "High temperature excitonic stimulated emission from ZnO epitaxial layers," Applied Physics Letters, vol. 73, no. 8, pp. 1038-1040, 1998.

[2] H. Cao, Y. G. Zhao, S. T. Ho, E. W. Seelig, Q. H. Wang, and R. P. Chang, "Random laser action in semiconductor powder," Physical Review Letters, vol. 82, no. 11, pp. 2278-2281, 1999.

[3] X. W. Sun, S. F. Yu, C. X. Xu, C. Yuen, B. J. Chen, and S. Li, "Room-temperature ultraviolet lasing from zinc oxide microtubes," Japanese Journal of Applied Physics, vol. 42, pp. L1229-L1231, 2003.

[4] M. H. Huang, S. Mao, H. Feick et al., "Room-temperature ultraviolet nanowire nanolasers," Science, vol. 292, no. 5523, pp. 1897-1899, 2001.

[5] C. Czekalla, C. Sturm, R. Schmidt-Grund, B. Cao, M. Lorenz, and M. Grundmann, "Whispering gallery mode lasing in zinc oxide microwires," Applied Physics Letters, vol. 92, no. 24, Article ID 241102, 2008.

[6] M. Ding, D. X. Zhao, B. Yao et al., "The ultraviolet laser from individual $\mathrm{ZnO}$ microwire with quadrate cross section," Optics Express, vol. 20, no. 13, pp. 13657-13662, 2012.

[7] B. S. Zou, R. B. Liu, F. F. Wang, A. L. Pan, L. Cao, and Z. L. Wang, "Lasing mechanism of $\mathrm{ZnO}$ nanowires/nanobelts at room temperature," The Journal of Physical Chemistry B, vol. 110, no. 26, pp. 12865-12873, 2006.
[8] D. Wang, H. W. Seo, C.-C. Tin et al., "Lasing in whispering gallery mode in $\mathrm{ZnO}$ nanonails," Journal of Applied Physics, vol. 99, no. 9, Article ID 093112, 2006.

[9] S. Okamoto, Y. Minowa, and M. Ashida, "White-light lasing in $\mathrm{ZnO}$ microspheres fabricated by laser ablation," in Oxide-Based Materials and Devices III, 82630K, vol. 8263 of Proceedings of SPIE, San Francisco, Calif, USA, January 2012.

[10] D. Yu, Y. Chen, B. Li et al., "Structural and lasing characteristics of ultrathin hexagonal $\mathrm{ZnO}$ nanodisks grown vertically on silicon-on-insulator substrates," Applied Physics Letters, vol. 91, no. 9, Article ID 091116, 2007.

[11] X. Liu, W. Fang, Y. Huang et al., "Optically pumped ultraviolet microdisk laser on a silicon substrate," Applied Physics Letters, vol. 84, no. 14, pp. 2488-2490, 2004.

[12] E. V. Chelnokov, N. M. Bityurin, and W. Marine, "Two-photon pumped zinc oxide random laser," in Proceedings of the Topical Problems of Nonlinear Wave Physics, vol. 5975 of Proceedings of SPIE, February 2006.

[13] G. P. Zhu, C. X. Xu, J. Zhu, C. G. Lv, and Y. P. Cui, "Twophoton excited whispering-gallery mode ultraviolet laser from an individual $\mathrm{ZnO}$ microneedle," Applied Physics Letters, vol. 94, no. 5, Article ID 051106, 2009.

[14] C. F. Zhang, F. Zhang, T. Xia et al., "Low-threshold two-photon pumped ZnO nanowire lasers," Optics Express, vol. 17, no. 10, pp. 7893-7900, 2009.

[15] J. Dai, C. X. Xu, and X. W. Sun, "Single-photon and threephoton absorption induced whispering-gallery mode lasing in ZnO micronails," Optics Communications, vol. 284, no. 16-17, pp. 4018-4021, 2011.

[16] G. P. Zhu, C. X. Xu, J. Zhu, and M. H. Wang, "Growth mechanism and multiphoton-induced photoluminescence of crownlike zinc oxide," Journal of Materials Science, vol. 46, no. 6, pp. 1877-1883, 2011.

[17] Z. K. Tang, M. Kawasaki, A. Ohtomo, H. Koinuma, and Y. Segawa, "Self-assembled $\mathrm{ZnO}$ nano-crystals and exciton lasing at room temperature," Journal of Crystal Growth, vol. 287, no. 1, pp. 169-179, 2006.

[18] T. Nobis and M. Grundmann, "Low-order optical whisperinggallery modes in hexagonal nanocavities," Physical Review A, vol. 72, no. 6, Article ID 063806, 2005.

[19] I. Braun, G. Ihlein, F. Laeri et al., "Hexagonal microlasers based on organic dyes in nanoporous crystals," Applied Physics B: Lasers and Optics, vol. 70, no. 3, pp. 335-343, 2000.

[20] Ü. Özgür, A. Teke, C. Liu, S. Cho, H. Morkoç, and H. O. Everitt, "Stimulated emission and time-resolved photoluminescence in rf-sputtered $\mathrm{ZnO}$ thin films," Applied Physics Letters, vol. 84, no. 17, pp. 3223-3225, 2004. 

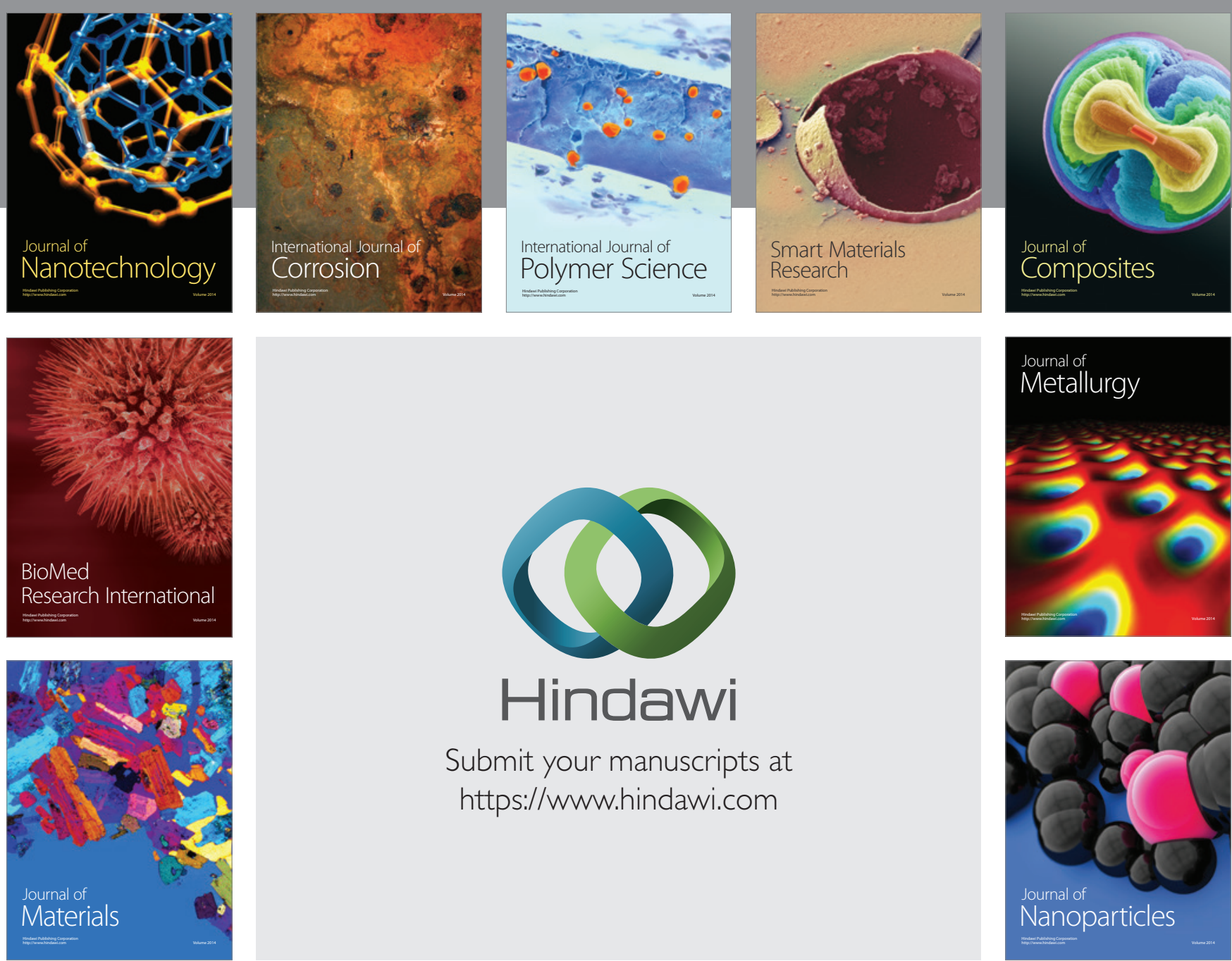

\section{Hindawi}

Submit your manuscripts at

https://www.hindawi.com

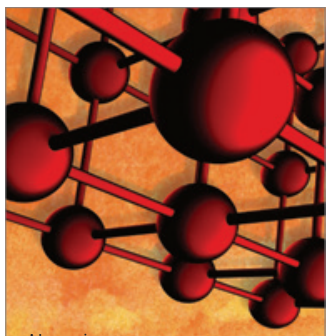

Materials Science and Engineering
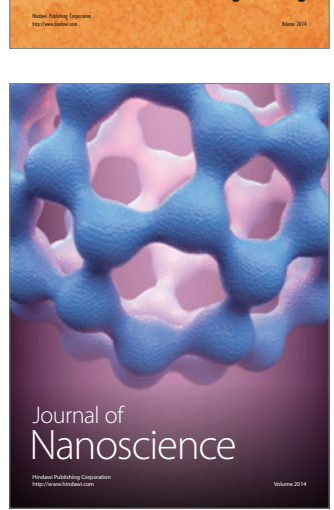
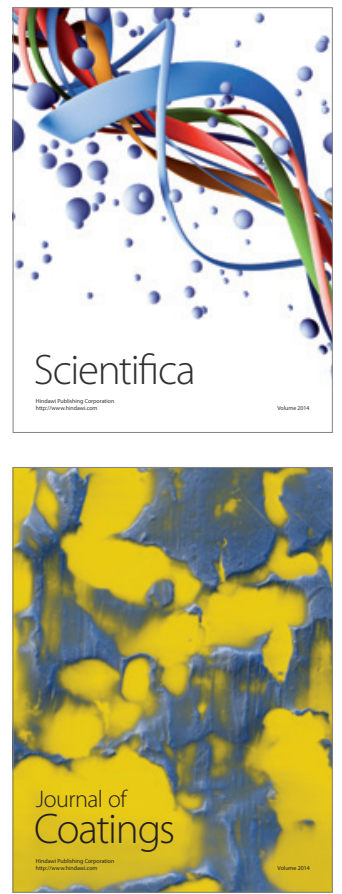
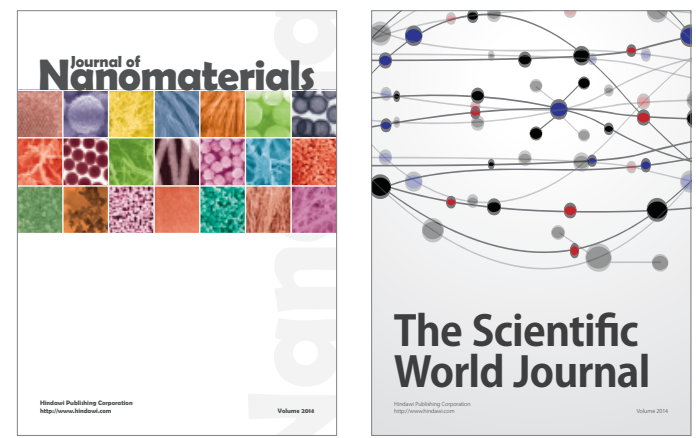

The Scientific World Journal
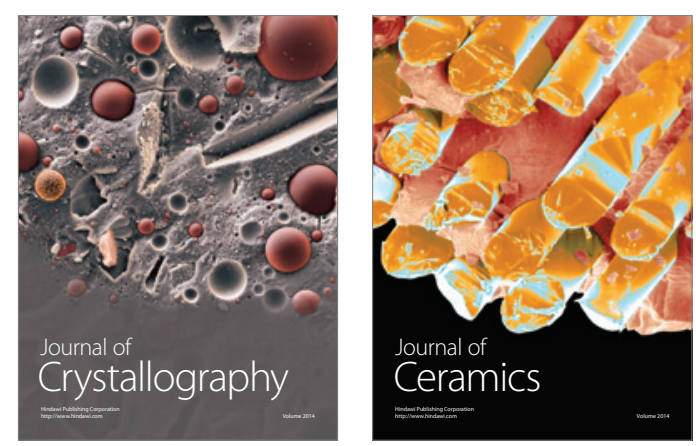
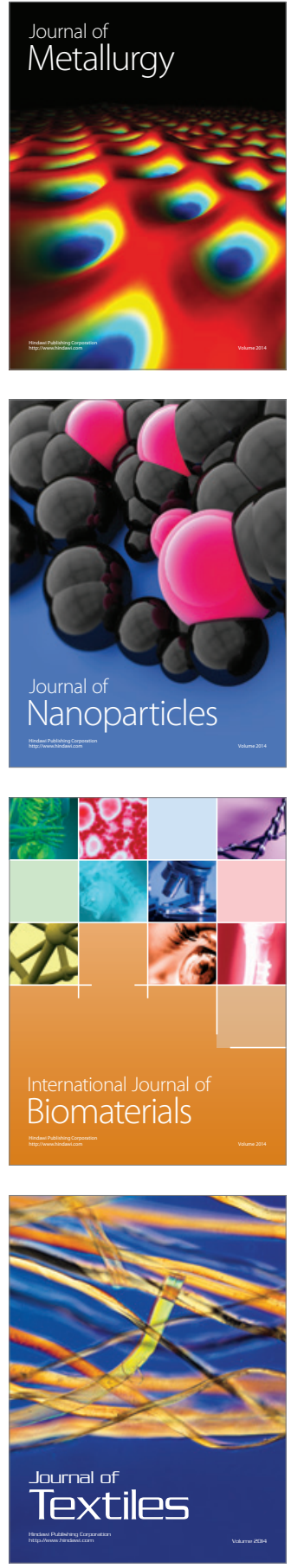